\title{
Osteomielitis crónica por Corynebacterium striatum en una adolescente
}

\author{
Claudia Beltrán-Arroyave, Alejandro Díaz-Díaz y Natalia Loaiza-Díaz
}

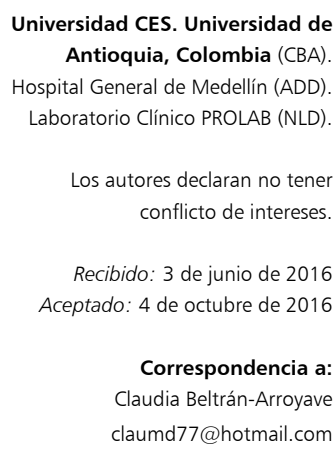

Universidad CES. Universidad de Antioquia, Colombia (CBA) Hospital General de Medellín (ADD) Laboratorio Clínico PROLAB (NLD)

Los autores declaran no tener conflicto de intereses.

Recibido: 3 de junio de 2016 Aceptado: 4 de octubre de 2016

Correspondencia a: Claudia Beltrán-Arroyave claumd77@hotmail.com

\section{Chronic osteomyelitis due to Corynebacterium striatum in a female adolescent}

A case of a girl with chronic posttraumatic osteomyelitis of the right tibia with microbiological isolation of Bacillus spp. by conventional methods and confirmation by mass spectrometry MALDI-TOF (matrix-assisted laser desorption/ionization time-of-flight mass) of Corynebacterium striatum is presented. Diagnostic methods, clinical manifestations, and resistance pattern of these infections are described.

Key words: Osteomyelitis, Corynebacterium, Corynebacterium infections

Palabras clave: Osteomielitis, Corynebacterium, infecciones por Corynebacterium.

\section{Introducción}

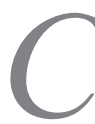
orynebacterium striatum ha sido considerado un microorganismo comensal de la piel y la mucosa nasal. Las infecciones por este microorganismo son poco comunes; sin embargo, en los últimos años se ha reconocido como un patógeno emergente no sólo en pacientes inmunocomprometidos sino también en inmunocompetentes. Estas infecciones se consideran un reto diagnóstico y terapéutico, en primer lugar porque la relación causal entre el sitio afectado y el aislamiento del microorganismo puede ser difícil de establecer dada su condición comensal, en segundo lugar, porque la identificación del microorganismo con las técnicas microbiológicas convencionales es difícil y finalmente porque este microorganismo puede expresar un alto perfil de resistencia a los antimicrobianos pudiendo dificultar el tratamiento.

\section{Caso clínico}

Niña de 13 años de edad, con antecedente de una fractura triplanar de tobillo derecho, tratada quirúrgicamente con material de osteosíntesis (MOS) retirado a los cuatro meses, cuya herida quirúrgica nunca cicatrizó. Consultó dos meses después del retiro del MOS por cambios inflamatorios en tobillo y salida de material purulento de mal olor. No presentaba signos de respuesta inflamatoria sistémica y había permanecido afebril. Recibió un curso de dicloxacilina empírica sin mejoría. Por este motivo se realizó un aseo y desbridamiento quirúrgico en el que se encontró un defecto de continuidad de aproximadamente $2,5 \mathrm{~cm} \mathrm{x} \mathrm{1,5} \mathrm{cm} \mathrm{en} \mathrm{la} \mathrm{región} \mathrm{maleolar} \mathrm{medial,} \mathrm{con} \mathrm{dre-}$ naje de un material óseo friable y necrótico. Se tomaron muestras de tejido para cultivo y se inició cefazolina intravenosa en forma empírica. La tinción de Gram mostró bacilos grampositivos y el cultivo demostró crecimiento de Bacillus spp., que fue considerado como contaminación y se continuó con el tratamiento prescrito. La paciente persistió con drenaje de secreción sero-hemática por la herida por lo que se realizó nuevo desbridamiento quirúrgico y se cambió la terapia a clindamicina intravenosa. En las muestras de tejido y hueso se obtuvo crecimiento de bacilos grampositivos identificados como Bacillus spp. sin posibilidad de identificación de especie ni patrón de susceptibilidad antimicrobiana por el método disponible (sistema VITEK 2). El antibiograma realizado por técnica de Kirby Bauer mostró resistencia a clindamicina, por lo que se decidió iniciar vancomicina intravenosa. La cepa finalmente fue identificada por la metodología MALDITOF MS (matrix-assisted laser desorption/ionization time-of-flight mass spectrometry) como Corynebacterium striatum. A los siete días de tratamiento con vancomicina la herida persistía con secreción. La resonancia magnética del tobillo (Figura 1) mostró una colección de tejidos blandos, edema perióseo, irregularidad de la cortical del hueso con un trayecto fistuloso a la piel. En la tercera intervención quirúrgica se corrigió la fístula fibrótica y se tomaron nuevas muestras para cultivos, en los cuales se obtuvo crecimiento del mismo microorganismo, identificado igualmente por técnica de MALDI-TOF como Corynebacterium striatum. Se realizó un antibiograma manual que demostró susceptibilidad a vancomicina, linezolid, daptomicina e imipenem, y resistencia a clindamicina, cefalosporinas, rifampicina, levofloxacina y cotrimoxazol. Las dosis de vancomicina se aumentaron hasta alcanzar niveles valle de $10 \mathrm{ug} / \mathrm{ml}$. La evolución clínica tras la última cirugía y el ajuste de la dosis fue satisfactoria, con término de la secreción y cicatrización completa de la 
herida. Se continuó tratamiento con vancomicina por 28 días y se completó tratamiento ambulatorio dos semanas más con linezolid oral. La paciente fue evaluada un mes después del egreso y la herida quirúrgica se encontró completamente sana, sin signos inflamatorios y con reactantes de fase aguda normales.

\section{Discusión}

Las bacterias del género Corynebacterium son bacilos grampositivos, forman parte de la microbiota de la piel y mucosas del ser humano y algunos animales. Se encuentran ampliamente distribuidas en el medio ambiente y pueden contaminar el medio hospitalario ${ }^{1}$. Son considerados microorganismos "fastidiosos" por su dificultad para crecer en los medios de cultivo y en las condiciones de incubación empleadas rutinariamente en el laboratorio. Se reconocen 73 especies dentro de las que se encuentra $C$. striatum. Este último ha sido considerado tradicionalmente un contaminante de muestras biológicas; sin embargo, se ha descrito como agente causal de endocarditis infecciosa, peritonitis, neumonías, infecciones de piel y tejidos blandos, infecciones osteoarticulares y meningitis, tanto en pacientes inmunocomprometidos como inmunocompetentes ${ }^{2,3}$. Su asociación con estas entidades clínicas es limitada, por su crecimiento lento y la dificultad para identificarlo con las metodologías microbiológicas convencionales ${ }^{2,4}$. Corynebacterium striatum tiene la capacidad de formar una biopelícula en dispositivos médicos, por lo que también se ha descrito en infecciones asociadas a catéter venoso central ${ }^{5,6}$.

Wong y cols. ${ }^{7}$, encontraron en una revisión de 17 meses, en el Hospital de Tai Sin en Hong Kong, 23 aislados en 17 pacientes, en el que utilizó el sistema API-Coryneâ para la identificación. Definieron que $C$. striatum fue el agente etiológico en nueve pacientes con neumonía, de los cuales sobrevivieron sólo la mitad aún con terapia antibacteriana apropiada. Los otros aislados fueron considerados contaminantes. También se ha identificado en infecciones de piel y tejidos blandos como el segundo agente etiológico de su especie precedido por C. amycolatum $^{8}$.

En relación a las infecciones osteoarticulares, como la de nuestra paciente, se encontraron escasos casos publicados en la literatura científica, todos en adultos. Existen dos reportes de artritis séptica en hombro y rodilla, además de una serie de casos de infección por Corynebacterium spp. en adultos sometidos a procedimientos ortopédicos, donde $C$. striatum fue aislado en $14 \%$ de los $\operatorname{casos}^{2,9,10}$.

Las infecciones por $C$. striatum también se han descrito en brotes nosocomiales en pacientes con factores de riesgo como hospitalización prolongada, enfermedad pulmonar crónica concomitante, uso reciente de antimicrobianos y realización de procedimientos diagnósticos invasores ${ }^{11}$.

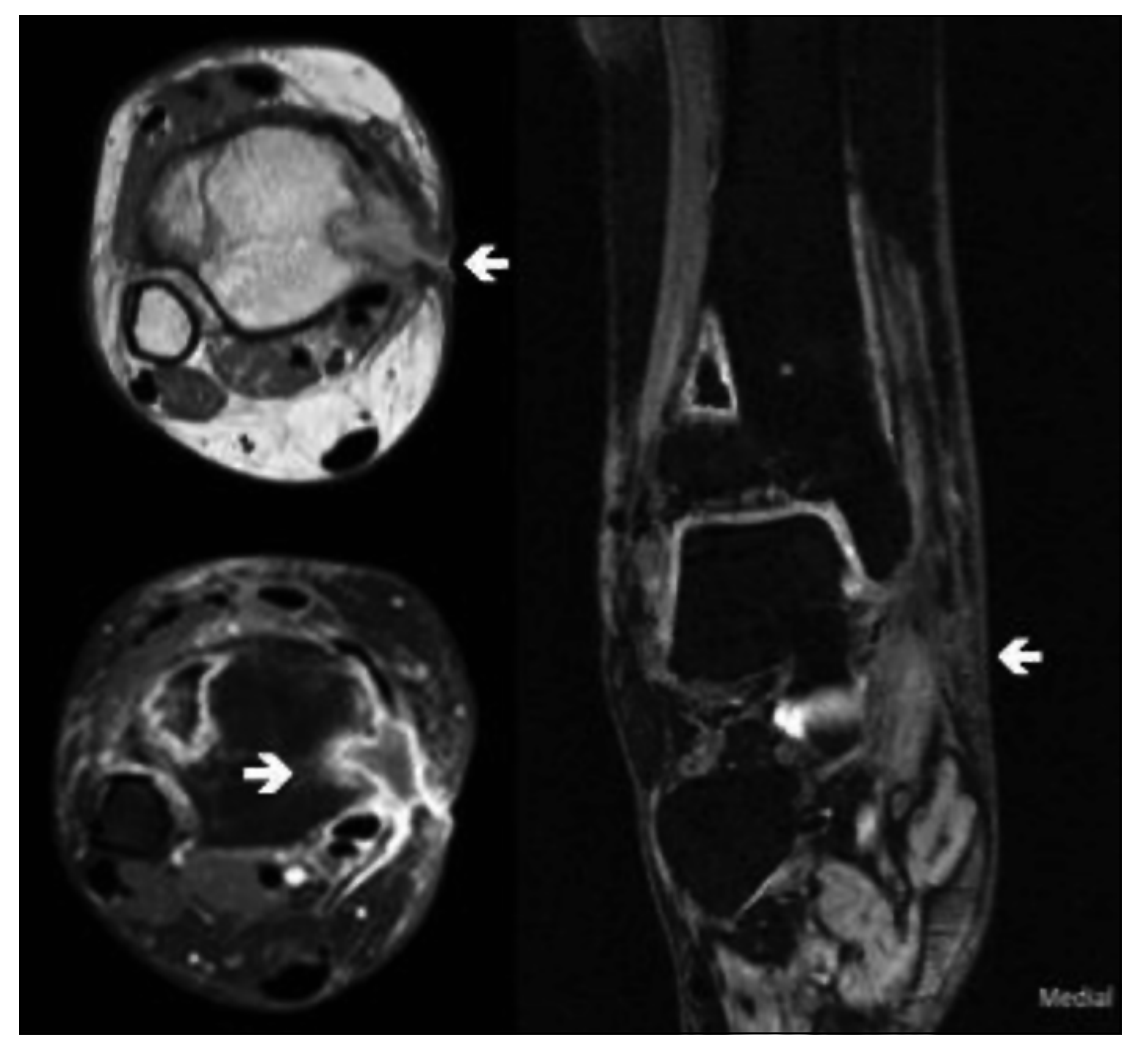

Figura 1. Resonancia magnética nuclear de tibia con gadolinio. Colección de tejidos blandos paraosteales de la cara medial en la unión metadiafisiaria de la tibia, con comunicación con el tejido celular subcutánea y la piel del compartimiento medial del tercio distal de la pierna. Realce y compromiso endosteal.

En un hospital universitario de Bélgica, durante el estudio de un brote en un período de ocho meses, se recuperaron 24 aislados de $C$. striatum en muestras de tracto respiratorio inferior de 10 pacientes. La identificación se realizó por la metodología MALDI-TOF MS y se realizó secuenciación genética. Tres de los pacientes se consideraron infectados por C. striatum ${ }^{11}$.

Existe escasa información en niños; una revisión de una serie de casos de pacientes oncológicos del hospital de St. Jude, E. U. A, entre 1992 y 2004, describió 33 pacientes con muestras clínicas con aislamiento de Corynebacterium spp., 17 de ellos (cuatro por C. striatum) fueron considerados infectados ${ }^{12}$.

Como se ha mencionado, la identificación de Corynebacterium spp. por técnicas convencionales suele ser deficiente, por lo que debe recurrirse a otras técnicas disponibles actualmente. MALDI-TOF MS tiene un buen rendimiento para identificar bacterias de este género (Score $>1.700$ el cual se considera confiable) ${ }^{13}$, por lo tanto, se considera una herramienta rápida y adecuada que ayuda a definir la terapia empírica de elección dado el perfil de multi-resistencia de estos microorganismos ${ }^{5}$. 
Otra herramienta es la secuenciación genética; sin embargo, su disponibilidad es mucho menor y de alto costo ${ }^{6}$. Lamentablemente en este caso no se pudo realizar la secuenciación de la cepa; sin embargo, se aisló el mismo agente en dos muestras tomadas con técnica aséptica en procedimientos quirúrgicos diferentes y se confirmó en las dos oportunidades la identificación de C. striatum por la técnica MALDI-TOF MS.

En cuanto a la susceptibilidad antimicrobiana, C. striatum es usualmente resistente a betalactámicos y sensible únicamente a vancomicina, linezolid y daptomicina ${ }^{1,2}$. Sin embargo, ya existen datos de resistencia adquirida para esta última, por lo cual no se recomienda usarla como monoterapia ${ }^{14}$. También se encuentran comunicaciones de aislados con sensibilidad únicamente a vancomicina. Remon y cols., reportaron que $16 \%$ de las cepas de $C$. striatum obtenidas de muestras respiratorias expresaban resistencia a dicho fármaco ${ }^{15}$. En el estudio de Wong y cols., en 11 de 17 cepas se documentó sensibilidad a vancomicina y todas eran resistentes a levofloxacina ${ }^{7}$. En la cepa aislada de nuestra paciente se determinó la susceptibilidad por difusión de disco (Kirby Bauer), metodología empleada en aquellos casos en los que los métodos automatizados que emplean concentración inhibitoria mínima (CIM) no ofrecen la posibilidad de realizar el perfil de susceptibilidad antibacteriana. También se podría realizar microdilución en caldo con antimicrobianos útiles contra cocáceas grampositivas ${ }^{10}$. El perfil de resistencia observado fue el usualmente encontrado en la literatura especializada, lo que se considera un valioso aporte a la hora de definir el tratamiento empírico cuando se obtenga crecimiento de bacilos grampositivos de difícil identificación, con alta sospecha clínica de $\operatorname{ser} C$. striatum como causante de infección.
Aunque existen escasos datos sobre la mortalidad global por Corynebacterium spp., se ha encontrado que en infecciones graves como endocarditis es de aproximadamente $45 \%$ y específicamente de $25 \%$ para infecciones por C. striatum ${ }^{4}$.

Se concluye con la presentación de este caso que $C$. striatum es un microorganismo emergente que debe ser considerado patógeno ante un paciente con infección clínica sin otro aislamiento microbiológico. Dada su difícil identificación por los métodos automatizados convencionales, la técnica MALDI-TOF MS representa una herramienta útil y disponible en nuestro medio para la identificación de este y otros microorganismos "fastidiosos" e infrecuentes. Las opciones de tratamiento son limitadas debido a los altos niveles de resistencia a varias de las familias de antimicrobianos disponibles. Se recomienda que ante aislamientos de bacilos grampositivos potencialmente causantes de un proceso infeccioso activo, se inicie vancomicina como terapia empírica, hasta disponer de los resultados de la identificación y las pruebas de susceptibilidad antibacteriana correspondientes.

\section{Resumen}

Se presenta el caso de una adolescente con osteomielitis crónica postraumática de la tibia derecha con aislamiento microbiológico por métodos convencionales de Bacillus spp. y confirmación por espectrometría de masas MALDI-TOF de Corynebacterium striatum, microorganismo considerado generalmente saprófito. Se describen las presentaciones clínicas de estas infecciones descritas en la literatura científica, la necesidad del diagnóstico etiológico por técnicas no convencionales y el patrón de resistencia comúnmente expresado.

\section{Referencias bibliográficas}

1.- Kim R, Reboli A C. Chapter 207: Other coryneform bacteria and rhodococci. En: Mandell, Douglas, and Bennett's principles and practice of infectious diseases. Bennett J E, Dolin R, Blaser M J, eds. Philadelphia. 8th Edition. 2015; 1194-215.

2.- $\quad$ Feced C, Alegre J, Ivorra J, Román J. Artritis séptica de hombro debida a Corynebacterium striatum. Carta al editor. Reumatol Clin 2013; 9: 383-5.

3.- Lee P P Ferguson D A, Sarubbi F A. Corynebacterium striatum: an underappreciated community and nosocomial pathogen. J Infect 2005; 50: 338-43.

4.- Boltin D, Katzir M, Bugoslavsky V, Yalashvili I, Brosh-Nissimov T, Fried M, et al. Corynebacterium striatum: a classic pathogen eluding diagnosis. Eur J Intern Med 2009; 20: e 49-52.

5.- Souza Cd, Faria Y D, Sant‘Anna L de O, Viana V G, Seabra S H, Souza M C, et al. Biofilm production by multiresistant Corynebacterium striatum associated with nosocomial outbreak. Mem Inst Oswaldo Cruz 2015; 110: 242-8.

6.- Yang H S, Kim Y J, Cho S Y, Shin E, Lee H J. Central venous catheter-related bloodstream infection by Corynebacterium striatum identified by $16 \mathrm{~S}$ rRNA and rpoB gene sequencing. Ann Lab Med 2015; 35 : 548-50.

7.- $\quad$ Wong K Y, Chan Y C, Wong C Y. Corynebacterium striatum as an emerging pathogen. J Hosp Infect 2010; 76: 371-2.

8.- Rudresh SM, Ravi G S, Alex A M, Mamatha K R, Sunitha L, Ramya K T. Non diphtheritic
Corynebacteria: an emerging nosocomial pathogen in skin and soft tissue infection. J Clin Diagn Res 2015; 9: DC19-21

9.- Westblade L F, Shams F, Duong S, Tariq O, Bulbin A, Klirsfeld D, et al. Septic arthritis of a native knee joint due to Corynebacterium striatum. J Clin Microbiol 2014; 52: 1786-8.

10.- Rizvi M, Khan F, Raza A, Shukla I, Sabir A B. Emergence of coryneforms in osteomyelitis and orthopaedic surgical site infections. Australas Med J 2011; 4: 412-7.

11.- Verroken A, Bauraing C, Deplano A, Bogaerts P, Huang D, Wauters G, et al. Epidemiological investigation of a nosocomial outbreak of multidrug-resistant Corynebacterium striatum at one Belgian university hospital. Clin Microbiol Infect 2014; 20: $44-50$. 
12.- Adderson E E, Boudreaux J W, Hayden R T Infections caused by coryneform bacteria in pediatric oncology patients. Pediatr Infect Dis J 2008; 27: 136-41.

13.- Alatoom A A, Cazanave C J, Cunningham S A, Ihde S M, Patel R. Identification of nondiphtheriae corynebacterium by use of matrix- assisted laser desorption ionization-time of flight mass spectrometry. J Clin Microbiol 2012; 50: $160-3$.

14.- Werth B J, Hahn W O, Butler-Wu S M, Rakita R M. Emergence of high-level daptomycin resistance in Corynebacterium striatum in two patients with left ventricular assist device infections. Microb Drug Resist 2016; 22: 233-7.

15.- Renom F, Gomila M, Garau M, Gallegos M D, Guerrero D, Lalucat J, et al. Respiratory infection by Corynebacterium striatum: epidemiological and clinical determinants. New Microbes New Infect 2014; 2: 106-14. 